
inventario de las dificultades auditivas de los niños en el hogar

The 'Child' Scale: alidation of the Children's Home Inventory for Listening Difficulties

(c) $\underset{\mathrm{BY}}{(1)}$
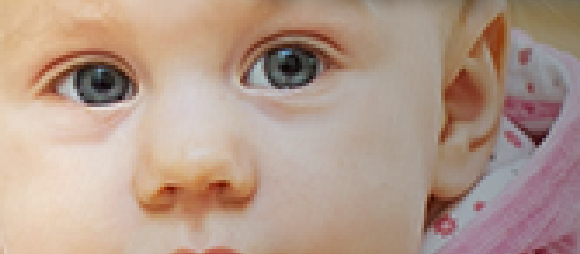

lana María Irurita Sánchez eydy Joanna Ramírez Tobar vianci Sepulveda Villamizar

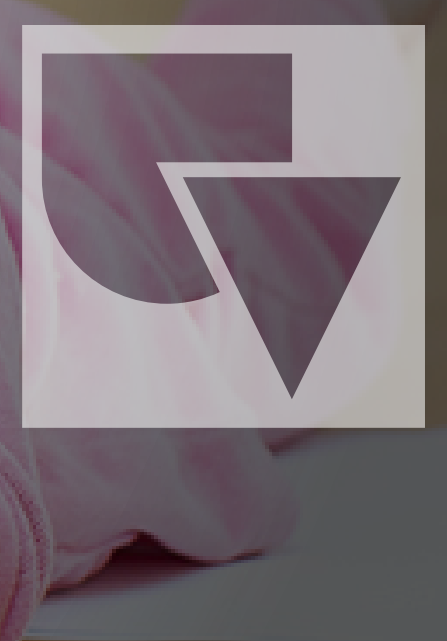

II

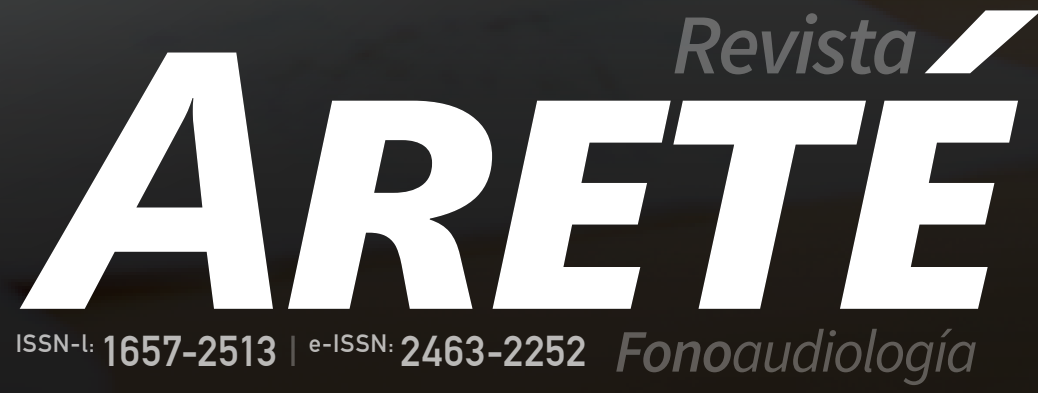




\section{ARETE \\ $1657-2513$}

ID:

1657-2513.art.17102

Title: The 'Child' Scale

Subtitle: Validation of the children's home inventory for listening difficulties

Título: Escala Child

Subtítulo: Validación del inventario de las dificultades auditivas de los niños en el hogar

Alt Title / Título alternativo:

[en]: Validation of the Child Scale, Children's Home Inventory for Listening Difficulties

[es]: Validación escala Child inventario de las dificultades auditivas de los niños en el hogar

Author (s) / Autor (es):

Irurita Sánchez, Ramírez Tobar, \& Sepulveda Villamizar

Keywords / Palabras Clave:

[en]: $\quad$ surveys and questionnaires; validation studies; hearing loss; hearing aids; audiology; communication

[es]

encuestas y cuestionarios; estudios de validación; pérdida auditiva; audífonos; audiología; comunicación

Submited: 2017-05-31

Acepted: 2017-11-10

\section{Diana María Irurita Sánchez, BHS sp}

Source | Filiacion:

Universidad del Valle

BIO:

Fonoaudióloga. Especialista en Audiología.

Docente Universidad del Valle. Audiologa en

Oír Audiología.

City | Ciudad:

Cali [Co]

e-mail:

diana_irurita@hotmail.com

\section{Resumen}

En Colombia, es común encontrar que el Fonoaudiólogo Especialista en Audiología, no lleva a cabo en su práctica clínica diaria un control estandarizado o riguroso de la funcionalidad de las ayudas auditivas en contexto, a partir del cual pueda identificar las necesidades comunicativas y/o mejoras en las habilidades auditivas de los niños necesarias dentro del ambiente de su hogar, que es el ambiente primario de aprendizaje. Por

esta razón se realizó un estudio para evaluar el grado de validez de la escala C.H.I.L.D. (Inventario de las Dificultades Auditivas de los Niños en el Hogar), y así determinar su aplicabilidad en población colombiana. Para esto se solicitó el concepto de nueve jueces expertos, quienes valoraron la pertinencia, suficiencia, claridad y coherencia/cohesión de contenido y sintaxis de los enunciados y las opciones de respuesta, a partir de las sugerencias proponer los respectivos ajustes

de la escala. Para el análisis de la información, se usaron dos medidas, el Índice Global de Acuerdo y el Índice de Acuerdo Kappa de Randolph (2005)

Se obtuvieron altos índices de concordancia para las preguntas y opciones de respuestas de

la escala, tanto en la versión de niños como la de padres. Se puede concluir que el proceso de validez de contenido efectuado fue eficiente para determinar su uso en Colombia.

Leydy Joanna Ramírez Tobar, ${ }^{\text {BHs sq }}$ ORCID: 0000-0002-6771-0635

$\mathrm{BIO}$ :

Fonoaudióloga. Especialista en Audiología. Audióloga en GAES Colombia S.A.S.

City | Ciudad:

Bogotá DC [Co]

e-mail:

ljoannart@gmail.com

\section{Abstract}

In Colombia, it is common to find that Audiologists, do not carry out in their daily clinical practice standardized or rigorous check-ups of hearing aids functionality in context, from which they can identify the communication needs and/or the improvements in auditory skills of children within the home environment, which is the primary learning environment. This research aims to evaluate the degree of validity of the C.H.I.L.D. scale (Children's Home Inventory for Listening Difficulties) to determine its applicability in the Colombian population. For this, nine expert judges were asked to assess the relevance, sufficiency, clarity and coherence/ cohesion of content and syntax of the questions and response options in terms of yes/no, and provide the respective adjustments of the scale taking into account the observations made by them. For the analysis of the information, two agreement measurement were used, the Global Index Agreement and Kappa Index Agreement of Randolph (2005). High concordance rates were obtained for the questions and response options for both children and parents scale's

versions. It can be concluded that the process of the validity of content performed was efficient to determine its use in Colombia.
Angela Vivianci Sepulveda Villamizar, внs sp BIO:

Fonoaudióloga. Especialista en Audiología. Fonoaudióloga en Centro Pediátrico Harker

City | Ciudad:

Bogotá DC [CO]

e-mail:

avivianci@gmail.com

Citar como:

Irurita Sánchez, D. M., Ramírez Tobar, L. J., \& Sepulveda Villamizar, A. V. (2017). Escala Child: Validación del inventario de las dificultades auditivas de los niños en el hogar. Areté issn-l:1657-2513, 17 (1), 9-14. Obtenido de: https://revistas. iberoamericana.edu.co/index.php/arete/article/view/973 


\title{
Escala Child Validación del inventario de las dificultades auditivas de los niños en el hogar
}

The 'Child' Scale: Validation of the Children's Home Inventory for Listening Difficulties

\author{
Diana María Irurita Sánchez \\ Leydy Joanna Ramírez Tobar \\ Angela Vivianci Sepulveda Villamizar
}

Al nacer el ser humano ya tiene un sistema periférico auditivo completamente maduro, por lo que la audición puede ser medida de manera cuantitativa y objetiva desde este mismo momento. Sin embargo, es la recopilación de múltiples experiencias y estímulos continuos a través de los sonidos lo que permite organizar un lenguaje estructurado por medio del cual se aprende y se comunica. Este proceso se desarrolla en el periodo denominado periodo crítico que es cuando se desarrollan las facultades neurológicas que permiten aprender el lenguaje (Rangel, 2007).

Cualquier tipo de alteración auditiva: permanente, temporal, fluctuante, progresiva, gradual, bilateral o unilateral presente durante este periodo crítico, hace que las bases para construir el lenguaje y los aprendizajes se desestabilicen lo cual puede generar retrasos en dichos procesos dependiendo del grado de pérdida auditiva y del momento en el que esta aparezca y de su persistencia a través del tiempo (Rangel, 2007). El déficit más evidente tiene lugar en la primera infancia al encontrarse una llamativa dificultad para adquirir de modo espontáneo y natural el lenguaje oral (Monsalve González \& Núñez Batalla, 2006). Además, se compromete seriamente el aprendizaje y uso correcto del lenguaje escrito, viéndose las personas sordas limitadas en su capacidad para adquirir conocimientos, aprender de modo autónomo y acceder a la información lingüística (CEAF, Comite de Expertos, 2005).

Actualmente existen diferentes estrategias para ayudar en el proceso de desarrollo del lenguaje de los niños con pérdidas auditivas, una de las opciones más usadas son los dispositivos médicos auditivos (implantes y audífonos). En niños de edades tempranas es difícil obtener su opinión subjetiva de la calidad de la adaptación (niveles de comodidad, molestia e inteligibilidad de las palabras) (Calvo \& Maggio, 2003). Por tanto, es necesario aplicar estrategias adicionales a las pruebas clínicas audiológicas, que permitan evaluar la funcionalidad de las ayudas auditivas en contexto.

Aunque existen muchas escalas que permiten evaluar la funcionalidad de las ayudas auditivas en contexto (Neira, y otros, 2009; Barcasnegras Cantillo, y otros, 2014); estas no se encuentran validadas para población colombiana, entre estas podemos encontrar la escala CHILD Children's Home Inventory for Listening Difficulties, en español conocida como Inventario de las Dificultades Auditivas de los Niños en el Hogar. Escala diseñada por Karen Anderson y la cual es apropiada para ser utilizada por el Fonoaudiólogo Especialista en Audiología con familias de niños usuarios de ayudas auditivas que tienen un nivel de desarrollo desde los tres años hasta los 12 años aproximadamente (Maggio, 2012).
Este estudio valido el contenido de la escala CHILD, con el propósito de que ésta pueda ser empleada por los Fonoaudiólogos Especialistas en Audiología de Colombia, dentro de la práctica clínica audiológica, como una herramienta habitual en la valoración y control de los pacientes con ayudas auditivas. Logrando mejores procesos de adaptación, al contar con un instrumento de evaluación con criterios de validez, eficacia y confiabilidad que no sólo evalúe el funcionamiento de la ayuda auditiva en ambiente clínico, sino también en contexto.

El objetivo entonces fue determinar el grado de validez de la escala CHILD, Para alcanzar este fin se convocó a un grupo de audiólogos a nivel nacional para que a través de su juicio y basados en sus conocimientos y experticia en la materia, contribuyeran a la verificación del instrumento, estableciendo la pertinencia, suficiencia, claridad y coherencia/cohesión de contenido y sintaxis de las preguntas y opciones de respuestas de la escala CHILD, de igual forma analizando la validez de contenido de la escala CHILD para posteriormente realizar los ajuste de contenido de la misma. 
Escala Child

Validación del inventario de las dificultades auditivas de los niños en el hogar

Materiales y Método

La presente investigación es de enfoque cuantitativo, alcance descriptivo y diseño no experimental transversal (Hernández Sampieri, Fernández Collado, \& Baptita Lucio, 2010). Su propósito consistió en evaluar la validez de una escala en términos de la pertinencia, suficiencia, claridad y coherencia/cohesión de contenido y sintaxis de las preguntas y opciones de respuestas mediante el juicio de expertos, en un momento único de la investigación. Para ello, se contó con la participación de 9 jueces expertos, Fonoaudiólogos Especialistas en Audiología, que tuvieran como mínimo 10 años de experiencia en el área y que actualmente trabajan en adaptación de audífonos, preferiblemente en niños.

Inicialmente, se solicitó la autorización para el uso de la escala a sus autores Anderson y Smaldin, y a quien realizó su adaptación al español Maggio De Maggi. Seguidamente, se solicitó la valoración de un grupo de jueces expertos para que determinaran la pertinencia, suficiencia, claridad y coherencia/ cohesión de contenido y sintaxis de las preguntas, y opciones de respuestas de la escala en términos de si-no. Para esto se proporcionó un instrumento, en el que se mostró el contenido de la escala, los criterios a evaluar con su respectivo instructivo.

Posteriormente, se analizaron los resultados, a través del índice de acuerdo Randolph (2014), considerando como mayoría mínima 5 de 4 respuestas afirmativas. Estas fueron promediadas mediante un paquete operativo estadístico para calificar la validez de cada uno de los ítems evaluados y, por ende, de la escala en general. Finalmente, se brindaron los respectivos ajustes a la escala teniendo en cuenta las observaciones de los expertos.

\section{Resultados}

En el presente estudio se usaron 2 medidas de acuerdo, las cuales son el Índice Global de Acuerdo y el Índice de Acuerdo Kappa de Randolph (2014), éste último es un índice de acuerdo corregido por el azar entre múltiples evaluadores e independiente de la distribución marginal. Estas características se ajustan a los intereses y la naturaleza del estudio.

Se calcularon 2 índices por cada tabla. El primero fue el Índice Global de Acuerdo, que es una medida general del porcentaje del acuerdo observado sin corrección por azar; su medición toma valores entre 0 y 1 , donde 0 indica $0 \%$ de acuerdo global observado y 1 indica 100\% de acuerdo global observado. El segundo índice fue el Índice de Acuerdo Kappa de Randolph, el cual toma valores entre -1 y 1, donde 1 indica 100\% de acuerdo más allá del azar, 0 indica $0 \%$ de acuerdo más allá del azar; es decir igual al azar, y medidas negativas indican porcentajes de acuerdo por debajo de lo que se esperaría por azar. Los índices de acuerdo (Tabla 1 y 2), tienen en cuenta las variables Pertinencia, Coherencia, Claridad, y Cohesión y Coherencia Sintáctica, lo anterior tanto para Preguntas en Padres, Opciones de Respuesta en Padres, Preguntas en Niños y Opciones de Respuesta en Niños. Además, se generó una tabla de acuerdo en donde se toma el valor 1 si cada juez evaluó con "SI" las variables Pertinencia y Coherencia y 0 en otro caso.
Tabla 1 Índice de acuerdo escala padres

\begin{tabular}{ccccc}
\multicolumn{2}{c}{\begin{tabular}{c} 
Variable \\
\multirow{2}{*}{ pertinencia }
\end{tabular}} & RK & 0.4444444 & 0.5333333 \\
& AG & 0.7222222 & 0.7666667 \\
\hline \multirow{2}{*}{ coherencia } & RK & 0.4888889 & 0.4888889 \\
& AG & 0.7444444 & 0.7444444 \\
\hline \multirow{2}{*}{ Claridad } & RK & 0.6148148 & -0.1111111 \\
& AG & 0.8074074 & 0.4444444 \\
\hline \multirow{2}{*}{ Cohesión y coherencia sintáctica } & RK & 0.2222222 & -0.1111111 \\
& AG & 0.6111111 & 0.4444444 \\
\hline \multirow{2}{*}{ General } & RK & 0.4222222 & 0.48889 \\
& AG & 0.7111111 & 0.744445
\end{tabular}

Tabla 2 Índice de acuerdo escala niños

\begin{tabular}{|c|c|c|c|}
\hline Variable & índice & pregunta & Respuesta \\
\hline \multirow{2}{*}{ Pertinencia } & RK & 0.4444444 & 0.5111111 \\
\hline & AG & 0.7222222 & 0.7555556 \\
\hline \multirow{2}{*}{ Coherencia } & RK & 0.4888889 & 0.2444444 \\
\hline & AG & 0.7444444 & 0.6222222 \\
\hline \multirow{2}{*}{ Claridad } & RK & 0.2362363 & -0.1111111 \\
\hline & AG & 0.6481481 & 0.4444444 \\
\hline \multirow{2}{*}{ Cohesión y coherencia sintáctica } & RK & 0.02362963 & -0.1111111 \\
\hline & $A G$ & 0.5148148 & 0.4444444 \\
\hline \multirow{2}{*}{ General } & RK & 0.4222222 & 0.244444 \\
\hline & $A G$ & 0.7111111 & 0.622222 \\
\hline
\end{tabular}

De acuerdo a los resultados obtenidos en el proceso investigativo se encontró: Pertinencia y Coherencia, índices que sugieren un grado de concordancia más allá del azar, indicando que hubo acuerdo entre los jueces al clasificar los ítems. Todos los porcentajes de validación estuvieron por encima del $\mathbf{7 8 \%}$, indicando alta tendencia en aceptación de la Pertinencia y Coherencia de cada una de las preguntas y opciones de respuesta de la escala. En el caso de Pertinencia para las preguntas en ambas versiones de la escala, 5 preguntas obtuvieron el $\mathbf{7 8 \%}$ de acuerdo y 10 el $\mathbf{8 9 \%}$ Para la Pertinencia en opciones de respuestas, en la versión de padres 1 opción obtuvo el $\mathbf{7 8 \%}$ y las otras 14 opciones el $\mathbf{8 8 \%}$; mientras en la versión de niños, 2 opciones el $\mathbf{7 8 \%}$ y 14 el $\mathbf{8 9 \%}$ Respecto a la Coherencia para las preguntas en la versión padres, 3 alcanzan el 78\% de acuerdo y 12 el $\mathbf{8 9 \%}$; entre tanto, en la versión de niños, 2 preguntas el $\mathbf{7 8 \%}$ y 13 el $\mathbf{8 9 \%}$. En las opciones de respuesta para la versión de padres 3 opciones el $\mathbf{7 8 \%}$ y en la versión de niños 1 el $\mathbf{8 9} \%$ y 14 el $\mathbf{7 8 \%}$.

Para los ítems de Claridad y Cohesión y Coherencia Sintáctica, se observa qué en las opciones de respuesta, tanto en la versión para niños como para padres, se obtuvieron índices Kappa de Randolph negativos; hecho que sugiere que los jueces tuvieron un acuerdo menor que el que se esperaría por azar. Caso contrario, en las preguntas se obtuvieron índices Kappa de Randolph positivos. Los porcentajes obtenidos de validación estuvieron por encima del $\mathbf{6 7 \%}$, mostrando aceptación en la calificación para indicar Claridad y Cohesión y Coherencia Sintáctica en las preguntas de ambas escalas. En las opciones de respuesta para padres y niños, no se observa una tendencia marcada en la aceptación de estos ítems. De acuerdo a estos resultados, se determinó que cada una de las preguntas de ambas versiones de la escala, cumple con los criterios de validación expuestos en los objetivos del estudio; caso contrario, para las opciones de respuesta, en las cuales los índices de acuerdo para Claridad y Cohesión y Coherencia Sintáctica se encontraron con valores inferiores a los esperados.

En cuanto a la Suficiencia se logró definir que el $\mathbf{8 8 \%}$ de los jueces determinaron que tanto las preguntas como las opciones de respuesta son suficientes para medir lo que se quiere cuantificar con las escalas. 


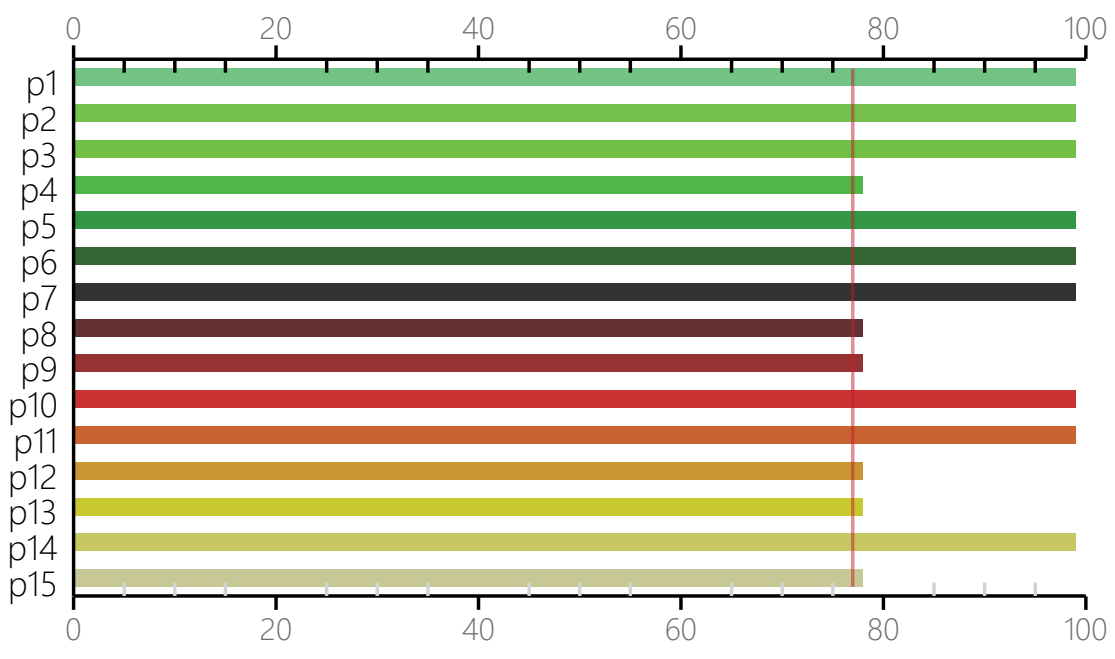

Gráfico 1 Porcentaje validación preguntas padres

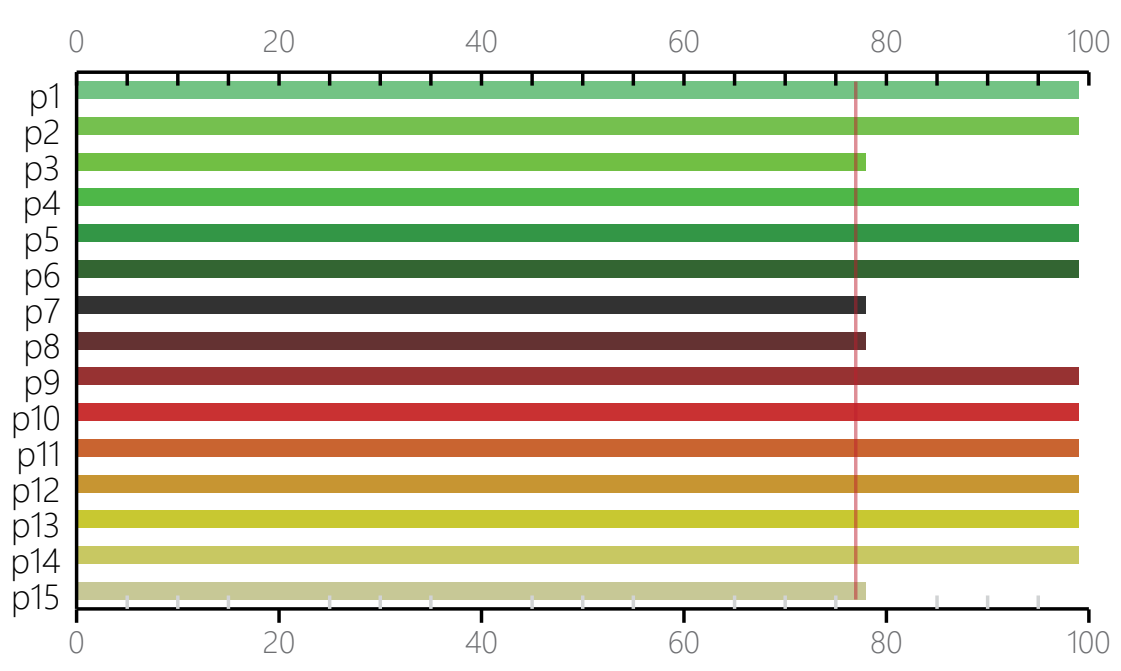

Gráfico 2 Porcentaje validación respuestas padres

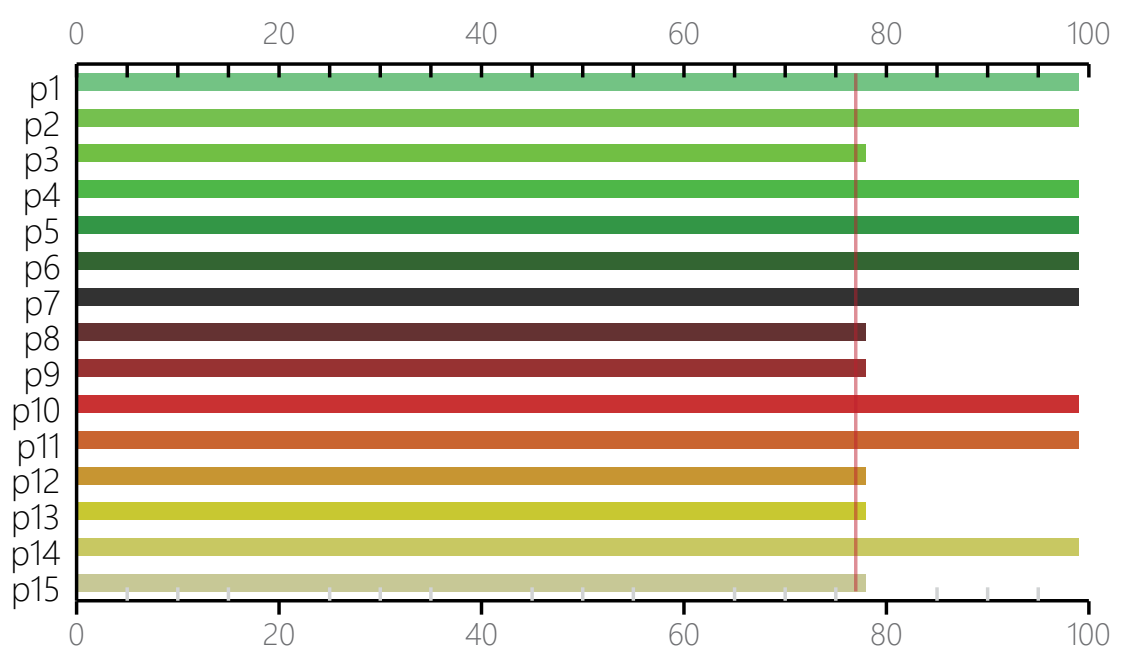

Gráfico 3 Porcentaje validación preguntas niños

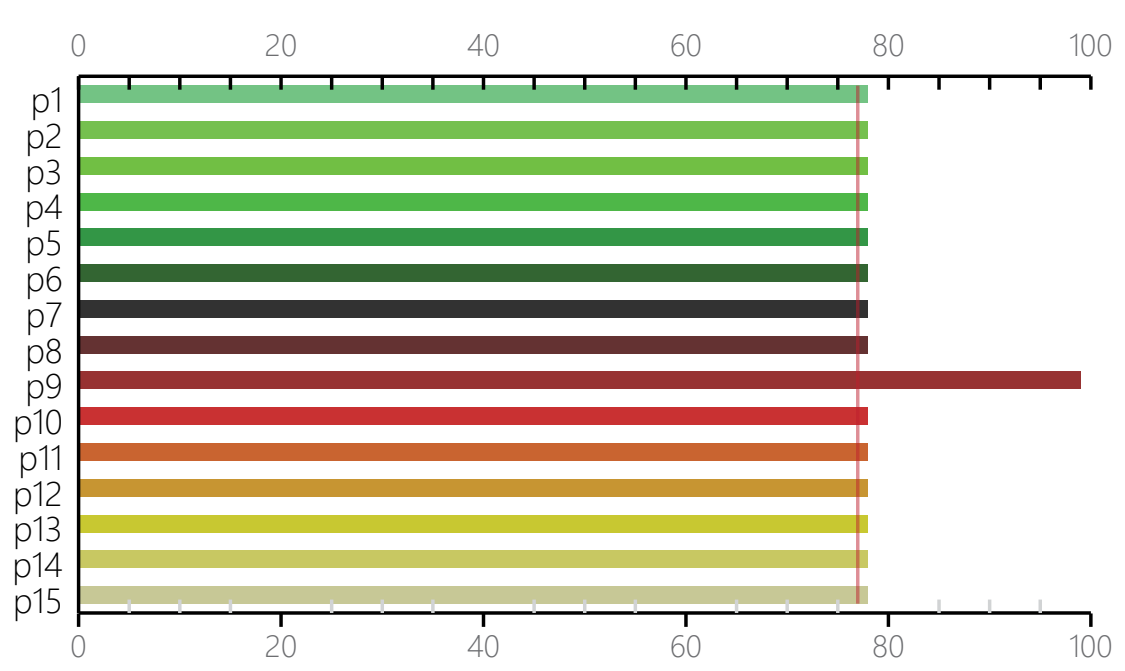

Gráfico 4 Porcentaje validación respuesta niños

En los Gráficos 1, 2, 3 y 4, se registran los datos generales de validación en donde se evidencian valores mayores a $\mathbf{7 7 \%}$. Lo que indica una alta tendencia en la aceptación de todas las preguntas y opciones de respuesta de ambas escalas.

\section{Discusión}

Es importante resaltar que el juicio o criterio de expertos es determinante en la etapa inicial de la construcción y validación de escalas en salud (Escobar Pérez \& Cuervo Martínez, 2008). En el estudio realizado tanto la versión de la escala CHILD para niños como para padres, obtuvieron un Índice Global de Acuerdo relativamente alto para los ítems de Pertinencia y Coherencia, hecho que indica un acuerdo general en la validación de la escala. Un proceso de validez requiere de la sistematización de tres fases:

1. Preparación, la cual incluye selección de expertos, preparación del instrumento y decisión de la vía de consulta

2. Consulta, esta incluye la ronda de consultas, el procesamiento estadístico y la realimentación

3. Consenso y reporte de resultados. (García Valdés \& Suárez Marín, 2013)

Las cuales se cumplieron en ésta investigación al:

1. Contar con 9 jueces expertos, convocados de acuerdo a criterios de selección y su experticia en el área, quienes diligenciaron el instrumento previamente elaborado.

2. Analizar la información obtenida a través de un software estadístico.

3. Obtener el consenso de índice de acuerdo de los ítems evaluados.

Lo anterior proporciona mayor confiabilidad, especificidad y validez de los resultados, por lo cual se puede determinar que el proceso de validez efectuado fue eficiente para determinar su uso en Colombia.

La validez de contenido es un proceso en el que se determina la estructura de la escala garantizando que ésta, por medio de sus ítems, abarque todos los dominios de la entidad que se quiere medir (Lamprea M. \& Gomez Restrepo, 2007). Como se observa en los resultados todas las preguntas y opciones de respuesta de ambas versiones de la escala son pertinentes, coherentes y suficientes, con respecto al propósito para el cual fue diseñada la misma. Se obtienen Índices Globales de Acuerdo altos, lo cual demuestra la concordancia en la calificación por parte de los expertos. La mayoría de los jueces refiere que la escala permite recolectar la información necesaria y acorde para determinar la funcionalidad auditiva de niños usuarios de ayudas auditivas en contexto. La escala evalúa situaciones cotidianas a las cuales se exponen los niños en su hogar, teniendo en cuenta los diferentes escenarios a partir de la relación señal-ruido: ambiente silencioso, ambiente moderadamente ruidoso y ambiente ruidoso.

La mayoría de los niños pequeños no pueden proporcionar información al Fonoaudiólogo Especialista en Audiología sobre sus audífonos; por tanto, la familia juega un papel fundamental en el proceso (CEAF, Comite de Expertos, 2005). Muchas mediciones utilizan a los padres como informadores porque ellos tienen la oportunidad de observar con más frecuencia en el ámbito familiar las conductas de interés. Existe una preocupación acerca de la capacidad de los padres para valorar las reacciones a los estímulos auditivos de sus hijos, por la manera en que se obtiene la información de los padres se considera una limitación inherente a las mediciones (Calvo \& Maggio, 2003). Por tanto, se considera importante que las escalas sean lo suficientemente claras para su comprensión y apropiado diligenciamiento por parte de los padres o todo aquel que la aplique, con el fin de obtener información válida, objetiva y útil.

A pesar que, en el estudio realizado, se obtuvo un alto porcentaje de acuerdo para la claridad, cohesión y coherencia 


\section{Escala Child}

Validación del inventario de las dificultades auditivas de los niños en el hogar

sintáctica de las preguntas de ambas versiones de la escala, no fue el caso para las opciones de respuesta, lo cual podría generar una respuesta inadecuada o condicionada. Por lo tanto, la escala requirió de modificaciones en las opciones de respuesta para su respectivo uso y validez.

De manera general, las modificaciones sugeridas por los jueces expertos fueron:

1. Reagrupar y organizar las preguntas de acuerdo al contexto auditivo: ambiente tranquilo con apoyo visual, sin apoyo visual, ambiente ruidoso, distancias, etc.

2. Para las preguntas que miden el nivel de escucha o comprensión con relación a la distancia e intensidad de una determinada fuente, especificar dichos criterios.

3. En las preguntas 3 y 6 , cambiar el vocabulario empleado "habitación propia" y "juguete ruidoso" por "habitación" y "objeto ruidoso" respectivamente, con el fin de evitar generar dificultad de aplicabilidad en estratos bajos, en caso que el niño no cuente con estos recursos.

4. En la pregunta 4 brindar diferentes opciones de fuente audiovisual, teniendo en cuenta los criterios de no contener subtítulos, ser visto por primera vez y estar acorde al nivel de desarrollo cognitivo del niño.

5. Para la pregunta 10 de la versión de la escala para padres, se cuestiona la viabilidad para comprobar la comprensión del niño en la interacción telefónica, si el padre desconoce lo dicho por la persona al otro lado del intercomunicador.

6. Se sugiere replantear la pregunta 8, puesto que al estar el niño apenas levantándose en la situación planteada, no estaría haciendo uso de sus ayudas auditivas.

Respecto a las opciones de respuesta, los jueces expertos refieren que no hay correspondencia entre la opción de respuesta con la pregunta, por lo que se podría modificar por opciones numéricas del 1 al 5, siendo:

1. No escucha o comprende nada, pierde todo el mensaje

2. A veces no escucha o comprende, pierde la mayor parte de la información

3. En ocasiones escucha y comprende, oye por lo menos la mitad de lo dicho

4. Escucha bien, oye bien, pero pierde parte de una palabra ocasional, aunque comprende todo

5. Escucha y comprende todo, oye cada palabra y comprende todo

De igual forma en la versión de la Escala para niños las preguntas deben ser más claras y concretas, con un lenguaje más sencillo, debido a que algunas preguntas están formuladas de forma compleja y podrían ser difíciles de comprender e interpretar por parte de los niños, Igualmente, en las opciones de respuesta para el niño puede ser difícil autocalificar su desempeño a partir de las opciones dadas. Se podría pensar en ajustar estas en términos de colores o símbolos para representar los diferentes niveles de dificultad o capacidad de desempeño.

El proceso efectuado permite determinar de forma eficiente y confiable la valides de la escala CHILD para su uso en Colombia, al obtener un Índice Global de Acuerdo relativamente alto, tanto para la versión de niños como la de padres, por parte de los jueces expertos que calificaron la misma.

Teniendo en cuenta el papel fundamental que juega la familia en el proceso de adaptación de audífonos en sus hijos, al brindar la información sobre su desempeño y comportamiento comunicativo, es importante que el Fonoaudiólogo Especialista en
Audiología cuente con escalas de validez que permitan evaluar y obtener esta información por parte de la familia y los niños, como es el caso de la escala CHILD

Es necesario continuar con el proceso de validación de la escala CHILD a través de la ejecución de una prueba piloto de la misma, empleando la escala con los ajustes realizados en el presente estudio; con el fin de determinar la validez de su uso en población infantil colombiana; y, por ende, considerarse instrumento evaluativo dentro del ámbito laboral del Fonoaudiólogo especialista en Audiología de Colombia.

\section{Referencias}

Barcasnegras Cantillo, D. P., Avilez Barros, E. P., Bermejo Colina, S., Ponnefz Grisales, V., Ortiz Ustariz, C. A., Ramírez Celedón, L. L., .. . Valencia Trujillo, M. (30 de Noviembre de 2014). Aplicación del li-srt en la evaluación de la discriminación de niños barranquilleros. (G. E. Rodríguez Jiménez, Ed.) Revista Areté, 14(1), 94 - 108.

Calvo, J., \& Maggio, M. (junio de 2003). Intervención audiologica centrada en la familia. Obtenido de marco referencial adaptación protesica infantil: http://phonak-pip.es/upfiles/files/A352.PDF

CEAF, Comite de Expertos. (Diciembre de 2005). Real patronado de discapacidad. Obtenido de la corrección protesica en niños: http:// www.fonoatriabielsa.com/doc/es-la-correccion-potesica-en niños.pdf

Escobar Pérez, J., \& Cuervo Martínez, Á. (2008). Validez de contenido y juicio de expertos: Una aproximación a su utilización. Avances de Medición. Avances en Medición, 6, 27-36. Obtenido de http://www.humanas. unal.edu.co/psicometria/files/7113/8574/5708/Articulo3_Juicio_de_ expertos_27-36.pdf

García Valdés, M., \& Suárez Marín, M. (Abril-Junio de 2013). El método Delphi para la consulta a expertos en la investigación científica. Rev Cubana Salud Pública, 39(2), 253-267.

Hernández Sampieri, R., Fernández Collado, C., \& Baptita Lucio, P. (2010). Metodología de Investigaciòn ${ }^{\circ}$ (Vol. 6). México: Mac Graw Hill.

Lamprea M., J. A., \& Gomez Restrepo, C. (2007). Validez en la evaluación de escalas. Revista Colombiana de Psiquiatría, 36(2), 340-348. Obtenido de Revista Colombiana de Psiquiatría: http://www.redalyc.org/ pdf/806/80636213.pdf

Maggio, M. (2012). Valoración de la capacidad funcional auditiva en los niños con deficiencias auditivas. Boletín AELFA, 34-40. Obtenido de Elsevier Doyma: http://elsevier.es

Monsalve González, A., \& Núñez Batalla, F. (07 de Junio de 2006). La importancia del diagnóstico e intervención temprana para el desarrollo de los niños sordos. Los programas de detección precoz de la hipoacusia. Psychosocial Intervention, 15(1), 7-28.

Neira, L. I., Walteros, D. P., Bueno, S. L., Ferrer, M. V., Sánchez, D. C., \& Sánchez N, L. A. (30 de Noviembre de 2009). Validez y confiabilidad de las listas li-srt en niños colombianos en edades de tres a cinco años. Revista Areté, 9(1), 73-84.

Randolph, J. (2014). Free-Marginal Multirater Kappa (multiraterkfree): An Alternative to Fleiss Fixed-Marginal Multirater Kappa. Conference Paper in Advances in Data Analysis and Classification, (págs. 4-21). Finlandia.

Rangel, P. (2007). Tamizaje Auditivo Infantil. En R. J. Antonio, Tratado de otología y audiología (págs. 681-683). Venezuela: Amolca. 